\title{
3D Face Measurement and Scanning Using Digital Close Range Photogrammetry: Evaluation of Different Solutions and Experimental Approaches
}

\author{
Luigi M. GALANTUCCI*, Fulvio LAVECCHIA, Gianluca PERCOCO \\ Politecnico di Bari, Italy
}

\begin{abstract}
This experimental research work was aimed to develop a 3D photographic method to be used in orthodontics diagnoses, having particular characteristics: precision of measurement, robustness of the method, speed of relief, ease of scanning, portability and low weight of the final system, low cost, non-invasive equipment.

Various solutions of the experimental set-up have been implemented for the non-contact detection and measurement of the face soft tissues.

This paper highlights the advantages and disadvantages of different set-up designed during the trials, comparing them to identify the best solution that meets the characteristics listed above.

Mainly two different types of photogrammetric approach were designed: the first one uses three cameras and projected grids in a dark environment; it is based on point triangulation. The second one uses 4 or 5 cameras and operates with applied on the background and on the face in ambient light; it is based also on the DSM methodology, and obtains more dense point clouds.

The systems were tested scanning the head of a mannequin and real human faces.

Within each type, several variants were tested to evaluate the differences, changing the conditions of projection, lighting, shooting. Moreover, the results for each approach were compared with those obtained by laser scanning (both on real face and on a mannequin), for which are already known the performances.

The system that best meets the requirements for diagnostic use in orthodontics proved to be the second one, in which coded targets applied directly on the face were used, shooting in ambient light, adopting digital photo sensors having 10 megapixels, and using 4-5 shots synchronized. The commercial software PhotoModeler was used for the CAD reconstruction of the face, and Geomagic for measures and comparisons.
\end{abstract}

Keywords: 3D face scanning, digital close range photogrammetry, orthodontics

\section{Introduction}

In recent decades, thanks to the availability of new detection and measurement systems, anthropometric studies that consider three-dimensional surfaces were developed [1], deepening the analysis of 3-D geometries and morphologies of the major outer tissues of the human body.

There are several methods of acquiring data on the form of a three-dimensional object [2].

The information on the shape of the human body has many medical applications [3]: for the collection of anthropometric data [4], but also for computer animation [5], for teleconferences and virtual reality [6], for forensic applications [7], for the sports training [8], for simulation testing of clothes [9] and to create products that take into account ergonomics [10].

Techniques of digitization of the human face must consider various aspects, such as realistic and accurate reproduction of the form obtained, the acquisition time for the model, the simplicity and cost of equipment.

For medical applications, non-contact methods are particularly interesting: the contact with the probe instruments during the measurement of soft tissues can deform the facial surface and thus can be a source of inaccuracy. Indirect methods, for which the interaction time with the patient is very limited, are less influenced by the behavior of the subject. Then, some measures, such as eyes, are difficult to obtain with direct methods without causing discomfort or injury to the patient [11]. The non-contact measuring methods do not provide the mechanical contact of the probe with the object: the interaction is implemented through electromagnetic fields, light or sound; the system obtains the information relating to the investigation by detecting energy transmitted or reflected from the body.

The Directive 97/43/EURATOM says that radiographic exposure is justified only when the patient management depends on the information obtained the radiograph. The optimal testing of a diagnostic record is to evaluate its consistency and validity. 
It is important to consider that problems as aesthetic treatments and surgery, orthodontics, malocclusions, cannot be consider always diseases: often they are not a biological abnormal state. So, that assessment of validity of a diagnostic record is not possible in the absence of the true state of disease. Thus, in studies where it can not be assessed the validity of the use of radiation, an alternative may be represented by comparisons of more non-invasive diagnostic tools.

In the medical field in general, and in particular orthodontic, the use of various investigative tools enhance the diagnostic clinical physical examination performed on the patient. Among these photographic examination it is crucial to analyze the current characteristics and temporal variations of soft tissues, spontaneous or therapeutically induced.

Many studies have been carried out on cephalometric measurements and on soft tissue obtainable by X-ray examinations, such as 3D CT and 3D Cone-Beam CT, instrumental tests that provide a threedimensional vision either of hard tissues either of the soft tissue of the patient [12], but that expose the patient to a moderate dose of x-ray.

The methods based on reflection uses images to provide useful spatial information in medicine, can be grouped into three main groups:

1. Measurements of surfaces and shapes of any body part (very small or large) for plastic surgery, reconstructive medicine, orthopedics, prosthetics, orthodontics and other dental specialties, dermatology and cosmetics;

2. Recording of the human body in motion (in which predominate walking studies);

3. Surgical measures in real time, to precisely position the surgical instruments and implants in the surgical theatre.

The solutions offered by major manufacturers vary in features, such as: technology (laser scanning projection of white light), building systems (fixed, CMM machines, robots, articulated arm), or type of measurement (surface scan, scan profile). The methods that detect the facial surfaces of 3D structures include laser surface scanning, moiré topography, contour analysis and detection of reflective markers with infrared cameras.

The 3D optical techniques for the acquisition of faces can be divided into:

1. 3D scanners [13]. The image acquisition is done by one or more laser beams of infrared light captured by mobile CCD camera. These methods are quite expensive, but also potentially very accurate. When a non static object is captured, such as a human face, there are significant limitations due to relatively high capture time (5 to $15 \mathrm{sec}$ ). The subject may move, causing errors in the final 3D model. 3D laser scanning has been widely studied, since the results give an opportunity to acquire high precision and complex shapes, but the cost of this equipment is very high, and the acquisition time requires (at least in the medical field) a great collaboration of the person concerned. 3D facial laser scanning systems (such as the Minolta Vivid 900 and 910) were used in medicine to detect and measure the morphology of the face and identify the location of anatomical landmarks on the virtual model, in order to assess its reliability in clinical perspective [3.14].

2. $3 D$ imaging systems based on structured light projection and analysis of moiré fringes: they require the projection of patterns on the face. The reflected images are acquired by CCD cameras, and then processed to determine the 3D coordinates of points [15].

3. Instantaneous 3D imaging systems based on photogrammetry: 3D information is obtained through the acquisition and comparison of several photographic images [16]. When using multiple synchronized cameras, they are ideally suited to digitize the human body, for insensitivity to slight movements of the body $[17,18]$.

The photogrammetry and laser scanning systems, thanks to the large number of anatomical points identified during the acquisition, have proved to be very useful in the morphological study of patients with facial asymmetry, facial clefts or other craniofacial disorders and dysmorphoses: in particular, these systems have proved valuable in providing a detailed morphological analysis of patients with complex facial deformities, since they avoid the distortion and magnification of typical radiographic images.

\section{3-D measurement using close range digital photogrammetry}

In last years begin to be available on the market some anthropometric equipment dedicated to the detection of the body surface, and in particular of the human face [2]. The major limitations of these optical systems are particularly cost, portability sometimes difficult, and often the difficulty to identify landmarks on the computer reconstruction of the facial surface [19].

Photogrammetry is on the contrary a method definitely cheaper, easy to use and requires a short acquisition time. The term photogrammetry means all those procedures that use photographic images of an object to obtain the position, shape and size. This technique allows to obtain the spatial position 
of the points of interest by photos and using a processing software for the image reconstruction [20]. The acquisition of information by photogrammetric techniques is very fast (4-6 ms), although subsequent data processing can still take a heavy human involvement.

The great opportunities offered by stereophotogrammetry are confirmed by the choice of the National Institutes of Health Clinical Center (NIDCR) [21] to use this method for a wide field survey in the United States, Brazil and Peru, on healthy volunteers and patients with craniofacial dysmorphoses.

\section{Experimental activity}

The authors have developed specific Research Projects of National Interest [18,22,23,24,25,26,27]. Through these projects low-cost scanning systems for photogrammetric diagnosis of facial and analysis of post-operative state have been designed. Three types of system were developed, based on a variable number ( 3 to 5 ) of high definition digital cameras (compact, SLR and prosumer). The systems operate under different conditions, which may include a projection of grid points (coded or not) in a dark environment, or the use of cold light lamps.

Regarding the number of points acquired, they were almost 1000 for the first configurations based on the triangulation points, and over 20,000 with the latest (based on matching areas), with the possibility of increasing more and more the point density, increasing the computing time. The point cloud, using Dense Surface Modeling algorithms, are dense, compact and precise $(0.5-0.6 \mathrm{~mm}$ on average, and for individual points measurement with coded target up to $0.03 \mathrm{~mm}$ on $X$ and $Y$ and $0.15 \mathrm{~mm}$ on $Z$, certainly more accurate than laser scanners, such as the Minolta Vivid 910i, which has an accuracy of $0: 38$ on $X, \pm 0: 31 \mathrm{~mm}$ on $Y$ and \pm 0.2 on $Z$ ).

\section{The photogrammetric scanning system}

The set for the photogrammetric scanning system is realized using cold light lamps, stands, tripods, flashes, cameras, computer remote control and analysis. In particular, two systems were made for photogrammetric measurement, which can use projected coded targets or coded target applied directly on the face of the patient. The first one is based on 3 Canon 40D, a pattern projector, a software for simultaneous control of the camera (shutter speed data, trigger, storing of images), and a photogrammetric software for the reconstruction of point clouds of faces (Fig. 1 and 2).

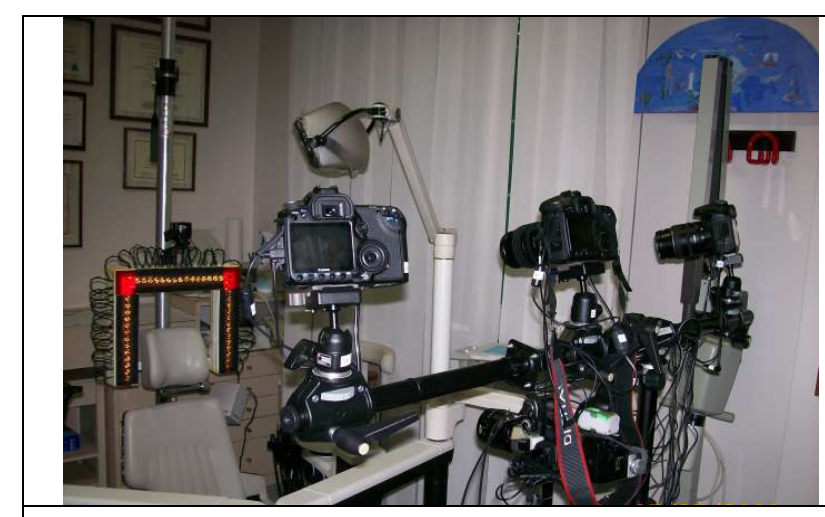

Fig. 1. photogrammetric set-up system with 3 cameras, projected grids and targets in a dark environment

Fig. 3. 4-Camera photogrammetric system set-up

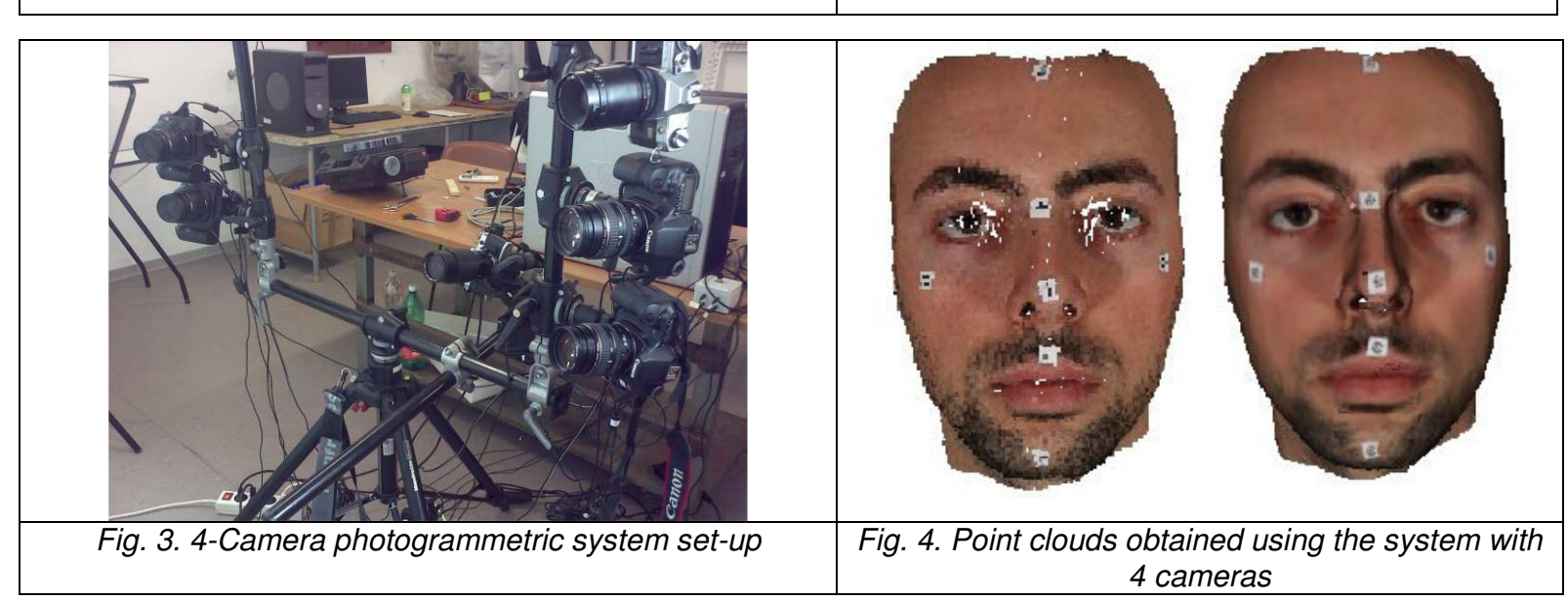

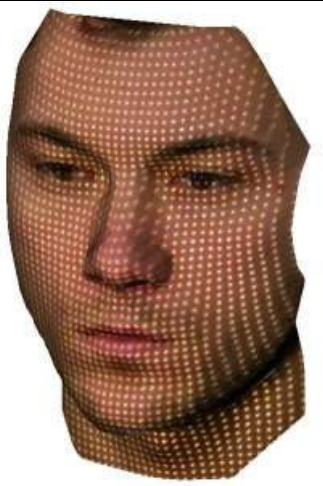

Fig. 2. Reconstruction of the face with the method to 3 cameras 
The 4-camera system (Fig. 3) consists of 4 high-definition digital SLR cameras, 2 flashes, 4 cold light lamps, computer that manages the process of simultaneous shooting to ensure cameras synchronization, and for the recording of the images. After a series of experiments the system was completely set and developed, and allows scanning and construction of clouds using either cold light lamps either external or internal flashes (Fig. 4).

Excellent results were obtained with the system having 5 cameras (Fig. 5). In this case the final configuration is one that involves the use of 3 external flashes, 2 couple of 2 cameras and 1 central camera. With the help of the central one, the cameras position calculation is optimized, and is improved the accuracy and the alignment of the clouds in prominent areas such as the nose (Fig. 6).

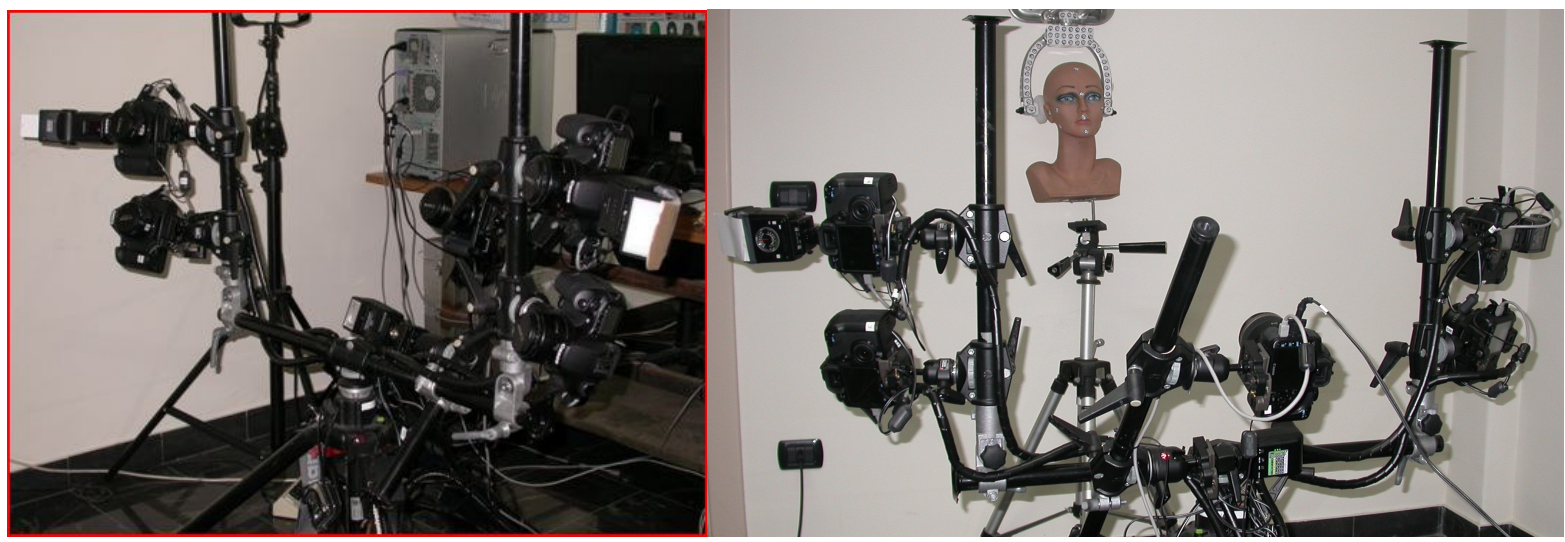

Fig. 5 Photogrammetric set-up with target on the background and applied on the face, using 5 cameras.

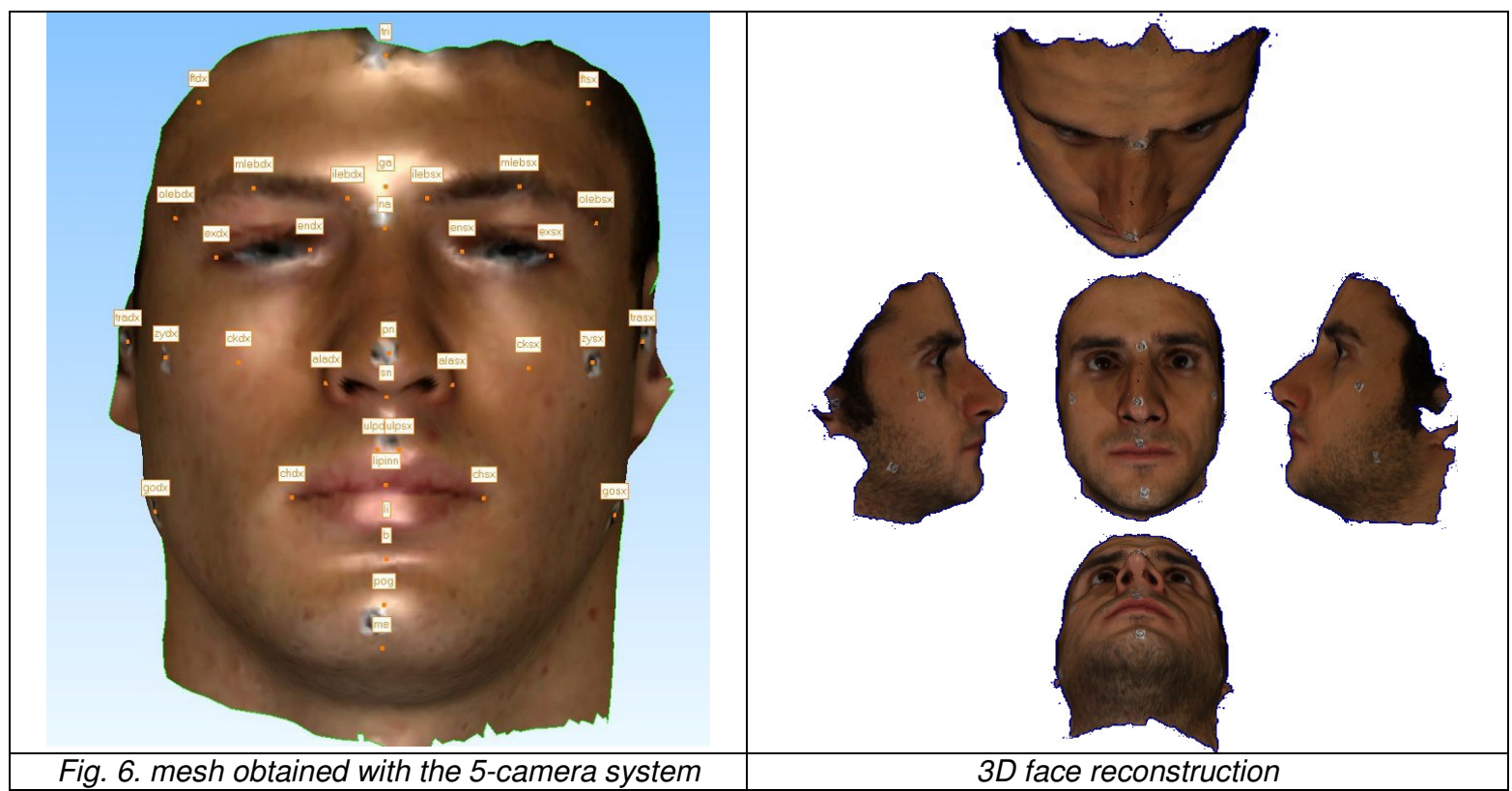

\section{Examples of reconstruction of the facial surfaces}

The point clouds were developed to identify landmarks relevant to the biometric analysis of the characteristics of the faces.

The first step is to align the origin of the three reference coordinate planes on virtual models of faces. The method is that proposed by Baik [28]. The Camper plane is firstly defined as the plane that passes through the wing of the nose and both the tragus points. The reference plane then is obtained from the axial rotation of the Camper plane of $7.5^{\circ}$ upwards, around the axis formed by both the tragus points (Fig. 7). 


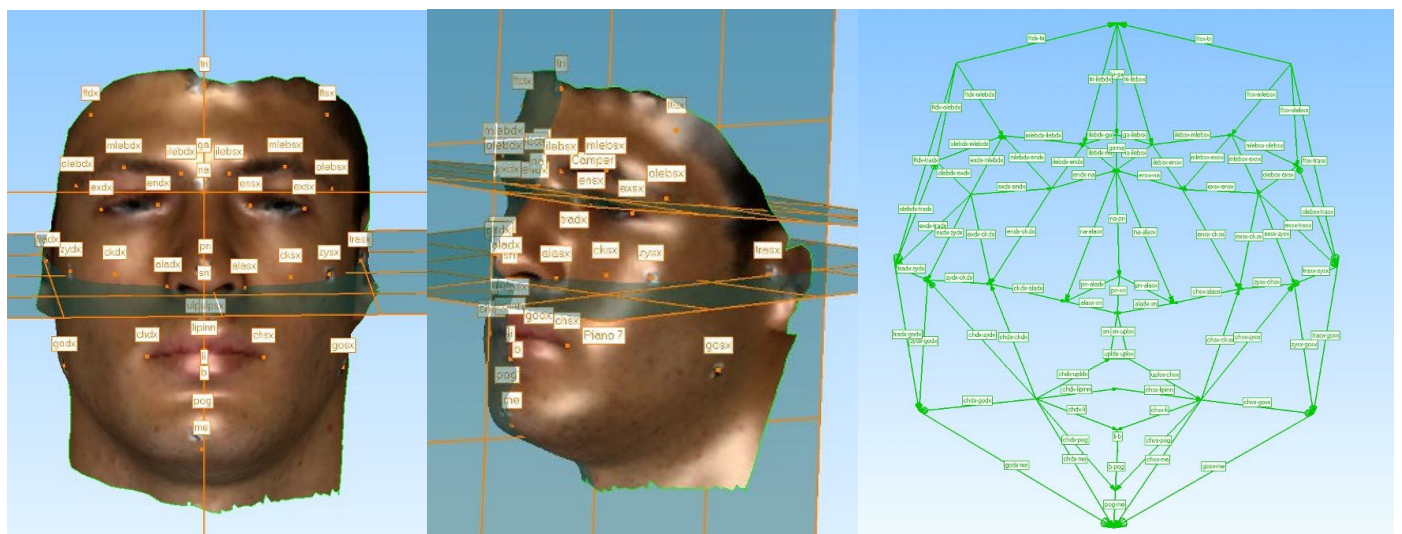

Fig. 7. Measure by method 3 coordinated plans and Landmarks on virtual models of faces and facial model using landmarks and polygons obtained between these points

The Axial reference plane is defined as plane passing through the Nasion ( $\mathrm{Na}$ ') soft tissue, parallel to the plane obtained by rotation of the Camper plane. The Sagittal reference plane is defined as a plane containing $\mathrm{Na}^{\prime}$ and the midpoint of the axis between both the tragus points, while perpendicular to the Axial reference plane. The Coronal reference plane is calculated as the plane through $\mathrm{Na}^{\prime}$, and simultaneously perpendicular to the axial and sagittal reference planes. These three reference planes define the new coordinate system whose origin is $\mathrm{Na}^{\prime}$ (zero point). All the landmarks coordinates are defined in this new coordinate system.

Some landmarks, such as the soft tissue Gonion fronto-temporal, and the Zygomatic point may be marked on the skin before scanning, to provide a better localization of the points in the 3D reconstruction.

A first objective was to obtain three-dimensional facial models through the measures outlined by obtaining for each face a specific calibration of these values (Fig 7).

\section{Analysis of symmetry}

Some preliminary tests of case studies of patients with facial asymmetry were carried out, using colour maps (Fig. 8).

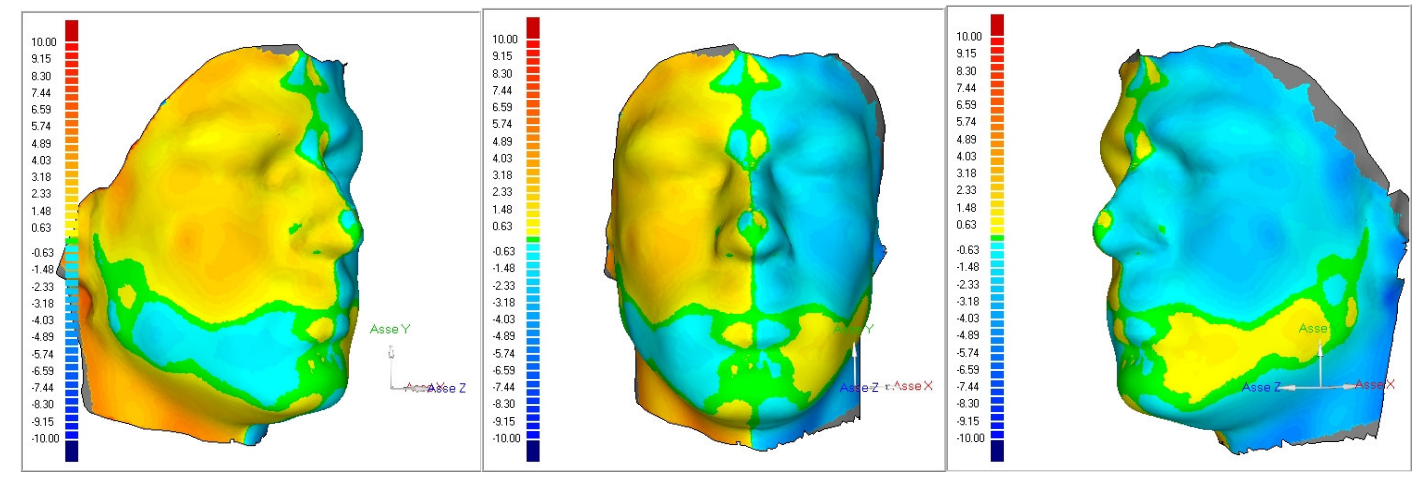

Fig. 8. Analysis of asymmetry with color maps

\section{Conclusions}

The anthropometry is an objective instrument for assessing the facial form and to detect changes over time, to diagnose genetic or acquired defects, for planning and evaluating surgical operations, to study normal and pathologic growth and to verify the results of the treatments.

The three-dimensional imaging diagnostic is an innovative approach in the field of medical disciplines, particularly dental, in which is emerging either in the clinical either in the research world. The image processing algorithms applied to facial images allow to develop and improve anthropometric applications by reducing the time required for examination, improve the reliability of measures, allowing to automatically detect clinically important distances and angles. These methods can be used to perform the analysis and comparison of the facial shape. Thanks to benefits of the images technology, the use of surface anthropometry has been extended to the full analysis of the face.

With the proposed methods it is possible to analyze the patient's facial type, the symmetry or asymmetry of a face, and evaluate the proportions between the different components of the facial 
profile. The 3D detection and measurement methods are an important diagnostic tool respect to the traditional 2D methods, because they could integrate three-dimensional assessment of soft tissue with the information obtained from two-dimensional cephalometric analysis.

The integration of data acquired using different technologies (CT, Cone Beam, Surface Scanning) may allow the clinician to customize more and more, and therefore improve the diagnostic and therapeutic approach to individual patients. Having a virtual 3D reconstruction of soft tissues rather than $2 \mathrm{D}$ photographic images of the face of patients, extends the capabilities of diagnostic and therapeutic clinical diagnostics, and may provide additional information on the dento-alveolar and/or skeletal disharmony hidden behind the profile and the face of the patient; in the therapeutic approach, it helps the clinician to investigate the aesthetic implications of dental-facial disharmonies, which are not a secondary aspect of treatment, as are often the real motivation of the patient to the treatment .

\section{Acknowledgements}

The present work has been funded by Italian Ministry for University and Research (MIUR), Project of Relevant National Interest PRIN 2007 "The Reverse Engineering for the non-invasive diagnostics in Orthodontics and Dentofacial Orthopedics: development of a system for automatic measurement of faces, based on photogrammetry".

\section{Bibliography}

1. Jones P., Rioux M. "Three-Dimensional Surface Anthropometry: Applications to the Human Body", Optics and Lasers in Engineering, pp. 89-117, NRC 40223, 1997.

2. Galantucci L.M.: "New challenges for reverse engineering in facial treatments: How can the new 3D non-invasive surface measures support diagnoses and cures?", Virtual and Physical Prototyping, Vol. 5, No. 1, March 2010, 1-10

3. Kovacs L., Zimmermann A. et al.: "Three-dimensional recording of the human face with a 3D laser scanner", Journal of Plastic, Reconstructive \& Aesthetic Surgery (2006) 59, $1193 \mathrm{e} 1202$

4. Lu J.M., Wang MJ.J.: "Automated anthropometric data collection using 3D whole body scanners", Expert Systems with Applications, (2008), 35(1-2): 407-414

5. Sitnik R., Kujawinska M., "Opto-Numerical Methods of Data Acquisition for Computer Graphics and Animation Systems", Three-dimensional Image Capture and Applications, Proc. of SPIE 3958, (2000), San Jose, USA, pp. 36-43,.

6. Shan et al.,: "Model-Based Bundle Adjustment with Application to Face Modeling", Proc. of the 8th Int. Conf. On Computer Vision (ICCV01) (2001), Vol. II, Vancouver, Canada, pp. 624-651,.

7. Larsen P.K., Hansen L., Simonsen E. B., Lynnerup, N.."Variability of bodily measures of normally dressed people using PhotoModeler Pro 5", Journal of Forensic Science, (2008), 53(6):1393-9.

8. Chong A.K., Croft H.G.: "A photogrammetric application in virtual sport training", The Photogrammetric Record, (2009), 24(125): 51-65.

9. Cho C. S., Park J. Y., Boeing A., Hingston P.: "An implementation of a garment-fitting simulation system using laser scanned 3D body data", Computers in Industry, In Press, Corrected Proof, Available online 17 April 2010

10. Lu J.M., Wang M.J.J., Mollard R.:"The effect of arm posture on the scan-derived measurements", Applied Ergonomics, (2010), 41(2): 236-241.

11. Swennen G.R.J., Schutyser F., Lemaitre A., Malevez C., De Mey A.: "Accuracy And Reliability Of 3-D Ct Versus 3-D Stereo Photogrammetry Based Facial Soft Tissue Analysis", Int. J. of Oral and Maxillofiacial Surgery, vol. 34, suppl.1 2005, pp 73

12. Swennen G.R.J., Schutyser F.: "Three-dimensional cephalometry: spiral multislice vs cone-beam computed tomography“, Am. J. of Orthodontics and Dentofacial Orthopedics, vol. 130, N. 3 - pp. 410-416

13. Okada, E.: "Three-Dimensional Facial Simulations and Measurements: Changes of Facial Contour and Units Associated with Facial Expression", Journal of Craniofacial Surgery, (2001),12, 2, pp. 167-174.

14. Kau C. H., Richmond S., Zhurov A.I., Knox J., Chestnutt I., Hartles F.,Playleg R.: "Reliability of measuring facial morphology with a 3-dimensional laser scanning system", Am J Orthod Dentofacial Orthop (2005);128:424-430

15. Yu W., Xu B.: "A portable stereo vision system for whole body surface imaging", Image and Vision Computing, (2010), 28(4): 605-613.

16. D’Apuzzo N.: "Surface measurement and surface tracking of human body parts from multi-image video sequences", ISPRS Journal of Photogrammetry and Remote Sensing, (2002), 56, 4, pp. 360-375. 
17. Ferrandes R., Galantucci L.M., Percoco G., "Optical Methods For Reverse Engineering Of Human Faces", 4th International CIRP 2004 Design Seminar, Session 6B pp. 1-12, Cairo, Egypt.

18. Deli R., Galantucci L. M., Di Gioia E., "Il Reverse Engineering per la diagnostica non invasiva in Ortognatodonzia: messa a punto di un sistema di misura dei volti automatico, basato sulla fotogrammetria", XXI Congresso Internazionale SIDO, 5-7 novembre 2009 Roma, p. 56-57

19. Sforza C.: "Analisi tridimensionale non invasiva della crescita craniofacciale in dentizione decidua e mista precoce", Ortognatodonzia Italiana vol. 13, 1-2006, pp. 53-62

20. Lane C., Harrell W., "Completing the 3-dimensional picture", American Journal of Orthodontics and Dentofacial Orthopedics, (2008), Vol 133, No 4,pp 612-620

21. "Stereo Photogrammetry Imaging in Normal Volunteers and Patients With Head and Facial Malformations", NIDCR, 9000 Rockville Pike, Bethesda, Maryland, 20892, USA PN: 05-D-0052 S. ID No: 050052; 05-D-0052, April 27, 2007 http://clinicalstudies.info.nih.gov/detail/A_2005-D0052.html

22. Galantucci L. M., Ferrandes R., Percoco G.: "Digital Photogrammetry for Facial Recognition", JCISE - Journal of Computing and Information Science in Engineering (2006) Vol. 6, 4, pp. 390396

23. Galantucci L.M., Percoco G., Dal Maso U., , Coded targets and hybrid grids for photogrammetric 3D digitisation of human faces, Virtual and Physical Prototyping, (2008) Vol. 3 issue 3, p: 167-176.

24. Di Gioia E., Deli R., Galantucci L.M., Percoco G., 2008, Reverse Engineering and photogrammetry for diagnostics in Orthodontics, Journal of Dental Research, Vol 87, Issue B, 1620

25. Di Gioia E., Deli R., Galantucci L. M., "Analisi con punti di repere sui tessuti molli di modelli 3D del viso con il metodo stereo fotogrammetrico 3D 3Cameras", XXI Congresso Internazionale SIDO, 5-7 novembre 2009 Roma, p. 57-58

26. Deli R., Di Gioia E., Galantucci L.M., Percoco G.: "Automated landmarks extraction for orthodontic measurement of faces using the 3 cameras photogrammetry methodology", Journal of Craniofacial Surgery, Volume 21, Number 1, January 2010, pp. 87-93

27. Galantucci L. M., Percoco G., Di Gioia E.: "Low cost 3D face scanning based on landmarks and photogrammetry: A new tool for a surface diagnosis in orthodontics", Intelligent Automation and Computer Engineering Series: Lecture Notes in Electrical Engineering, Vol. 52, Ao, Sio-long; Castillo, Oscar; Huang, Xu (Eds.) 2009, Springer, ISBN: 978-90-481-3516-5

28. Baik H., Jeon J., Leeb H., , Facial soft-tissue analysis of Korean adults with normal occlusion using a 3-dimensional laser scanner, American Journal of Orthodontics and Dentofacial Orthopedics, (2007), Vol 133, No 4,pp 612-620. 